\title{
High Efficiency Monolithic Photodetectors for Integrated Optoelectronics in the Near Infrared
}

\author{
Ali K. Okyay, M. Cengiz Onbasli, Burcu Ercan \\ Department of Electrical and Electronics Engineering, Bilkent University, Ankara, 06800, Turkey \\ (email: aokyay@ee.bilkent.edu.tr) \\ Hyun-Yong Yu, Shen Ren, David A. B. Miller, Krishna C. Saraswat \\ Department of Electrical Engineering, Stanford University, Stanford, CA 94305, USA \\ Ammar M. Nayfeh \\ Department of Electrical Engineering, San Jose State University, San Jose, CA 95192, USA
}

\begin{abstract}
Monolithic Germanium photodetectors integrated on Si with external efficiency up to $68 \%$ at $1550 \mathrm{~nm}$ and low dark current density $3.2 \mathrm{~mA} / \mathrm{cm}^{2}$ are demonstrated. The absorption edge red shifted by $47 \mathrm{~nm}$ corresponding to bandgap energy reduced by $24 \mathrm{meV}$.
\end{abstract}

Due to its inherent large indirect $(\sim 1.1 \mathrm{eV})$ and direct $(\sim 3.4 \mathrm{eV})$ bandgap energies, Silicon (Si) is near-infrared-blind for practical applications. InGaAs based optical detectors dominate today's NIR applications, but the high materials and integration costs prevent this market from growing. Germanium $(\mathrm{Ge})$ has been emerging as a low-cost solution to overcome the spectral limit of Si photodetectors (PDs) in transceivers and imagers. This interest is multiplied by Ge compatibility with Si CMOS technology, prompting researchers to focus on developing techniques for integration of $\mathrm{SiGe}$ and $\mathrm{Ge} \mathrm{PDs}$ on $\mathrm{Si}$ [1-3]. Ge growth on $\mathrm{Si}$ is hampered by the large lattice mismatch (4.2\%). This results in islanding and misfit dislocations terminating at the film surface as threading dislocations. The presence of defects in the film degrades detector performance by reducing carrier lifetime (hence lowering the efficiency) and increasing generation-recombination rate (hence increasing current leakage and noise). We report high efficiency monolithic Ge based PIN and MSM optical detectors for integration with Si CMOS using a recently developed technique, Multiple Hydrogen Annealing for Heteroepitaxy (MHAH) [4,5], for growing high quality thick heteroepitaxial Ge layers on Si.

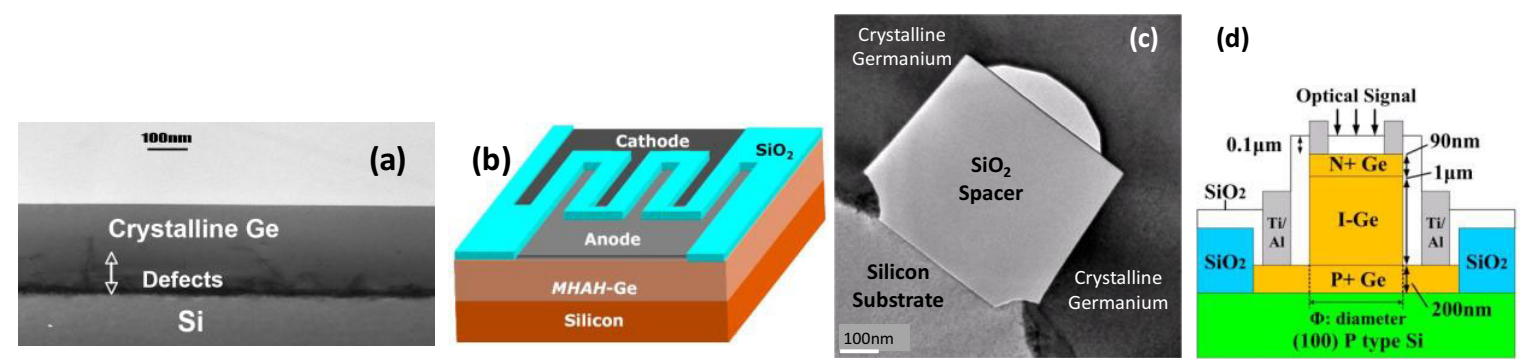

Fig. 1. (a) Cross-sectional TEM image of heteroepitaxial-Ge layer on Si grown by the MHAH method. (b) Cross section of MSM-PD fabricated on blanket Ge-on-Si. (c) Cross-sectional TEM image of selective area grown Ge. (d) Schematic of PIN-PD fabricated on selective grown Ge.

Ge layers obtained with this technique exhibit very low surface roughness $(2.9 \mathrm{~nm})$ and $50 \times$ reduction in dislocation density. Fig. 1(a) presents a cross sectional TEM image of a two step $M H A H$ growth that yielded a $\sim 400 \mathrm{~nm}$ Ge layer. Near the surface the dislocation density is very low $\left(\sim 10^{7} \mathrm{~cm}^{-2}\right)$. Fig. 1(b) shows the schematic of interdigitated MSM-PDs fabricated on such layers. It is desired to have control on specific location(s) Ge layers are grown for high density integration. We achieved this by selective area growth by patterning a $\mathrm{SiO}_{2}$ film on $\mathrm{Si}$ substrate to define growth windows with $\mathrm{SiO}_{2}$ spacers. Fig. 1(c) is a cross sectional TEM image from such grown Ge layer and $\mathrm{SiO}_{2}$ spacer that bounds the film. The side walls of the $\mathrm{SiO}_{2}$ spacer provide termination for threading dislocations emanating from the Si-Ge interface. The resulting crystal quality is very high. Fig. 1(d) is the schematic of PIN-PDs fabricated on Ge mesas grown by this method.

The dark current density $\left(J_{d a r k}\right)$ of the photodiode not only indicates the material quality but also determines optical receiver sensitivity [6]. Decent Schottky Ti-Ge contact was obtained on blanket grown films by currentvoltage $(I-V)$ characteristics as shown in Fig. 2(a), confirming the low defect density of the MHAH-Ge substrates. Fig. 2(b) shows the dark $I-V$ characteristics of photodiodes with various mesa radii fabricated on selective area grown Ge films, yielding $J_{\text {dark }}$ as $3.2 \mathrm{~mA} / \mathrm{cm}^{2}$, one of the lowest reported $J_{\text {dark }}$ values among the Ge PIN-PDs [7].

Detector photocurrent vs input optical power and responsivity vs reverse bias is plotted in Fig. 2(c) and 2(d), respectively. We observe responsivities $(\mathfrak{R})$ of $0.76 \mathrm{~A} / \mathrm{W}$ and $0.67 \mathrm{~A} / \mathrm{W}$ under $1 \mathrm{~V}$ reverse bias for MSM and PIN PDs, respectively. This corresponds to $61 \%$ and $53 \%$ external quantum efficiencies $(\eta)$, respectively. The highest $\Re$ of $0.85 \mathrm{~A} / \mathrm{W}$, corresponding to $\eta \sim 68 \%$ was observed from a detector with $5 \mu \mathrm{m}$ electrode width and spacing. Under 
similar conditions, the theoretical maximum collection efficiency is $88 \%$ without accounting for reflections from the surface. The residual strain is determined by Raman spectra measurements, yielding $0.141 \%$ and $0.204 \%$ for selective and blanket grown Ge films, respectively. The extracted tensile strain values in both cases arise from the difference in thermal expansion coefficients between $\mathrm{Ge}$ and $\mathrm{Si}$. The lower tensile strain value in the selectively grown Ge compared to bulk grown $\mathrm{Ge}$ is attributed to the $\mathrm{Ge}$ confinement by $\mathrm{SiO}_{2}$, which has compressive strain.

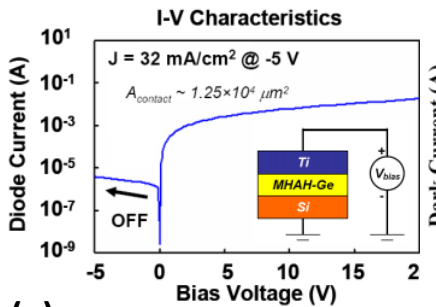

(a)

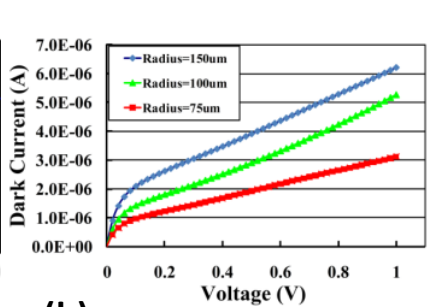

(b)

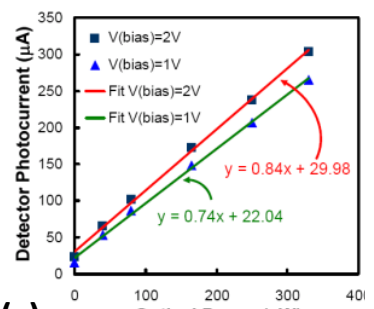

(c)

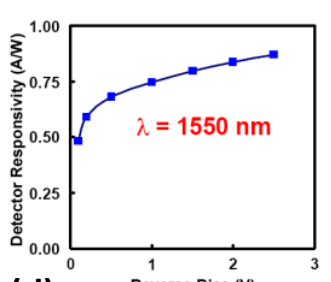

(d)

Fig. 2. (a) I-V characteristics of Ti-Ge Schottky diode on blanket MHAH-Ge. (b) Measured $I_{\text {dark }}$ for PIN photodiodes with various radii.

Ge film complies with the Si substrate and builds tensile strain which in turn induces bandgap shrinkage in the Ge layer [8]. Spectral measurements verified $\sim 24 \mathrm{meV}$ bandgap shrinkage which translates into $\sim 47 \mathrm{~nm}$ red shift in the absorption edge in addition to enhanced absorption efficiency, as depicted in Fig. 3(a) for blanket grown films. Detectors were built on bulk Ge wafers for reference purposes. Absorption coefficient values extracted from responsivity of PIN detectors on selective grown films is plotted in Fig. 3(b). Higher strain in the blanket grown films results in a larger red shift and smaller bandgap energy compared to selectively grown films.
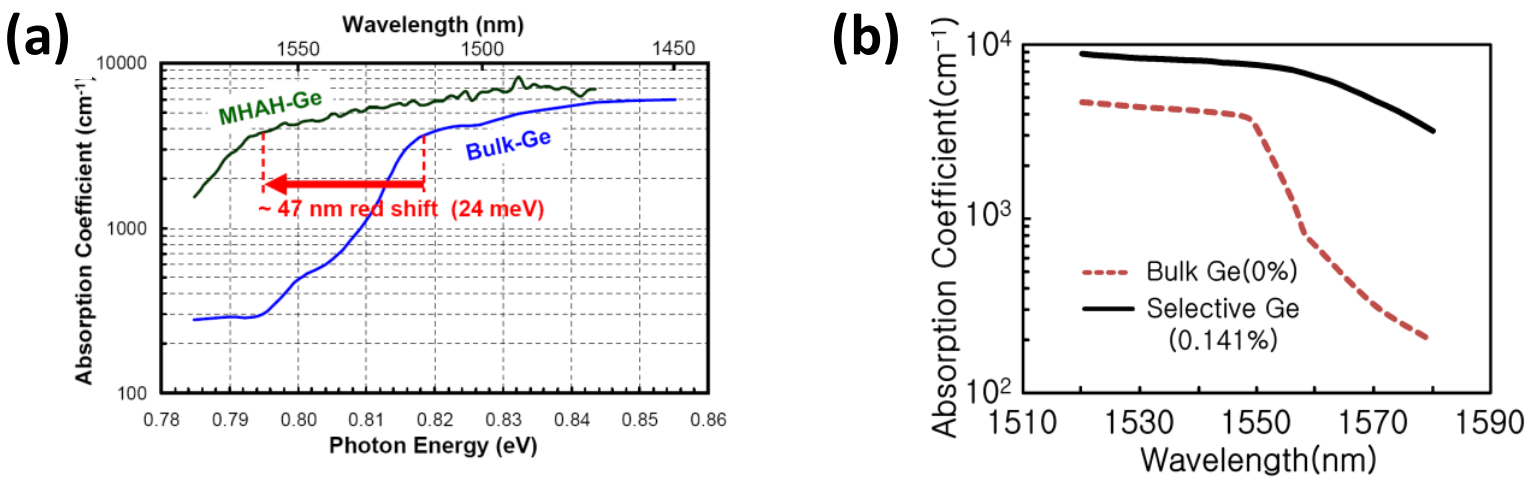

Fig. 3. Experimental absorption coefficient vs photon energy for (a) blanket grown and (b) selective area grown Ge-on-Si.

In summary, we demonstrate Ge PDs with $J_{\text {dark }}$ as low as $3.2 \mathrm{~mA} / \mathrm{cm}^{2}$ and $\eta \sim 68 \%$ at $1550 \mathrm{~nm}$ integrated directly on Si using a novel technique that allows growth of high quality heteroepitaxial-Ge layers on Si. The grown films are under tensile strain resulting in enhanced efficiency in the near infrared and a significant red shift in the absorption edge.

\section{References}

[1] S. Luryi, A. Kastalsky, and J. C. Bean, "New infrared detector on a silicon chip," IEEE Trans. Elect. Dev., vol. ED-31, 1135, 1984.

[2] L. Colace, G. Masini, G. Assanto, H. C. Luan, K. Wada, L. C. Kimerling, "Efficient high-speed near-infrared Ge photodetectors integrated on Si substrates," App. Phys. Lett., 76, 10, 1231, 2000.

[3] S. Fama, L. Colace, G. Masini, G. Assanto, H. C. Luan, "High performance germanium-on-silicon detectors for optical communications," App. Phys. Lett., 81, 4, 586, 2002.

[4] A. Nayfeh, C. O. Chui, T. Yonehara and K. C. Saraswat, "A Method to Grow Heteroepitaxial-Ge on Si: Multiple Hydrogen Annealing for Heteroepitaxy (MHAH)," MRS Spring 2005, San Fransisco, CA, 2005.

[5] H. Y. Yu, Jin-Hong Park, A. K. Okyay, and K. C. Saraswat, "Defect Reduction of Ge on Si by Selective Growth and Hydrogen Annealing," 214th ECS Meeting PRIME 2008, Paper 2466, Honolulu, HI, 2008.

[6] L. M. Giovane, H. C. Luan, A. M. Agarwal and L. C. Kimerling, "Correlation between leakage current density and threading dislocation density in SiGe p-i-n diodes grown on relaxed graded buffer layers," App. Phys. Lett. 78, 541, 2001.

[7] T. H. Loh, H. S. Nguyen, R. Murthy, M. B. Yu, W. Y. Loh, G. Q. Lo, N. Balasubramanian, and D. L. Kwong, "Selective epitaxial germanium on silicon-on-insulator high speed photodetectors using low-temperature ultrathin Si0.8Ge0.2 buffer," App. Phys. Lett. 91, 73503, 2007.

[8] Y. Ishikawa, K. Wada, D. D. Cannon, J. Liu, H. Luan, and L. C. Kimerling "Strain-induced band gap shrinkage in Ge grown on Si substrate," App. Phys. Lett., 82, 2044, 2003. 